\title{
Single Donor-Acceptor Pairs as a Tool for Studying Conformational Dynamics of Proteins and Other Macromolecules
}

\section{I.S. Osad'ko ${ }^{a}$}

Institute for spectroscopy of RAS, Russia, 142190, Moscow, Troitsk, Fizicheskaya 5

\begin{abstract}
Influence of triplet states in donor and acceptor molecules on function $E\left(R_{\mathrm{DA}}\right)$ describing dependence of FRET efficiency $\mathrm{E}$ on inter-dye distance $R_{\mathrm{DA}}$ is considered. Formula for $E\left(R_{\mathrm{DA}}\right)$ differs from conventional equation used widely in practice and it shows that triplet states hampers energy transfer in D-A pair, considerably.
\end{abstract}

Keywords: single donor-acceptor pair, efficiency of energy transfer.

In a few years after beginning of single molecule investigation it became clear that single donoracceptor (D-A) pairs attached to macromolecules can be used to study conformational dynamics of these macromolecules. Indeed, value of inter-dye distance $R_{\mathrm{DA}}$ between $\mathrm{D}$ - and A-molecules will change if conformation of the macromolecule is changed as Fig.1 shows.

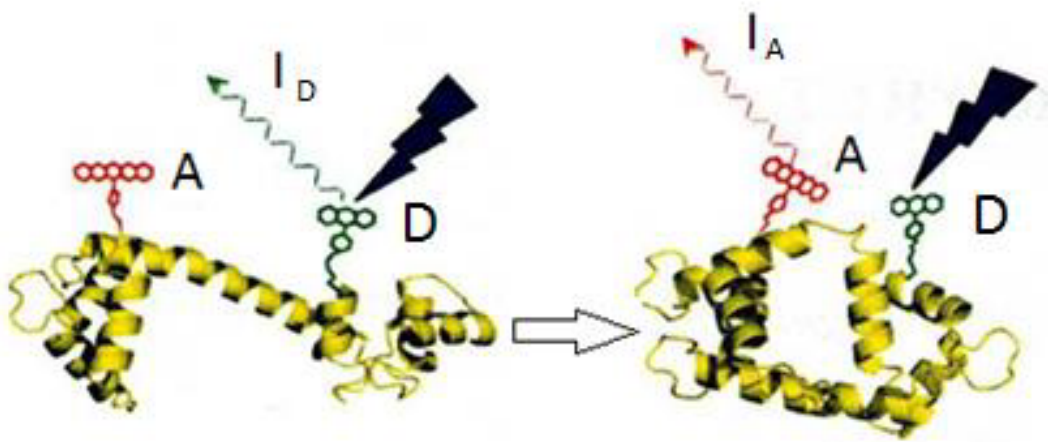

Figure 1. Appearance of acceptor fluorescence after change of the conformation of the protein molecule with attached single D-A pair.

Intensity $I_{\mathrm{A}}$ of A-fluorescence and intensity $I_{\mathrm{D}}$ of D-fluorescence will fluctuate in phase with fluctuations of inter-dye distance $R_{\mathrm{DA}}$. Therefore, by measuring fluctuations of the efficiency $E\left(R_{\mathrm{DA}}\right)=$ $I_{\mathrm{A}} /\left(I_{\mathrm{A}}+I_{\mathrm{D}}\right)$ we can find fluctuations of inter-dye distance $R_{\mathrm{DA}}$. However, it can be done only if we know shape of the function $E\left(R_{\mathrm{DA}}\right)$ for the average efficiency of the energy transfer. In huge number of experimental works the following formula is used:

${ }^{\text {a }}$ Corresponding author: osadko@isan.troitsk.ru 


$$
E\left(R_{\mathrm{DA}}\right)=\left[1+\left(R_{\mathrm{DA}} / R_{\mathrm{F}}\right)^{6}\right]^{-1}
$$

Here $R_{\mathrm{F}}$ is the Förster radius. In accordance with eq. (1) full energy transfer is possible if $E\left(R_{\mathrm{DA}}\right)$ approaches zero. It should be noted that eq.(1) was derived for the case in which D- and A-molecule have no "dark" states, i.e. excited states without light emission. However, organic molecules used in capacity of the donor and the acceptor molecule have triplet states and triplet states result in blinking fluorescence with on and off intervals. If we take into account existence of triplet states expression for the energy transfer takes the following form [1]:

$$
E\left(R_{\mathrm{DA}}\right)=\left[\Lambda+\left(R_{\mathrm{DA}} / R_{\mathrm{F}}\right)^{6}\right]^{-1} .
$$

Here $\Lambda>1$ is expressed solely via average durations $\tau_{\text {off }}$ and $\tau_{\text {on }}$ of off and on intervals. In accordance with eq.(2) full energy transfer is impossible between DA pairs with effective triplet states. However, at $\tau_{\text {off }} / \tau_{\text {on }}<<1$ we have $\Lambda \sim 1$, i.e. triplet states don't hamper energy transfer.

\section{Reference}

1. I.S. Osad'ko, J. Chem. Phys. 142, 1251021 (2015) 